\title{
Strong Correlations in Highly Electron-Doped Zn(II)-DNA Complexes
}

\author{
Aleš Omerzu, ${ }^{1}$ Bernarda Anželak, ${ }^{2}$ Iztok Turel, ${ }^{2}$ Janez Štrancar, ${ }^{1}$ Anton Potočnik, ${ }^{1}$ Denis Arčon, ${ }^{1}$ Iztok Arčon,,${ }^{1,3}$ \\ Dragan Mihailović, ${ }^{1}$ and Hiroshi Matsui ${ }^{4}$ \\ ${ }^{1}$ Jozef Stefan Institute, Jamova 39, 1000 Ljubljana, Slovenia \\ ${ }^{2}$ Faculty of Chemistry and Chemical Technology, University of Ljubljana, Aškerčeva 5, 1000 Ljubljana, Slovenia \\ ${ }^{3}$ University of Nova Gorica, Vipavska 13, SI-5000 Nova Gorica, Slovenia \\ ${ }^{4}$ Physics Department, Graduate School of Science, Tohoku University, Sendai 980-8578, Japan
}

(Received 7 August 2009; published 15 April 2010)

\begin{abstract}
We present spectroscopic measurements on the first example of a chemically electron-doped metalDNA complex. We show that a doping level as high as the stoichiometric limit of one electron per base pair can be achieved. The doped unpaired electrons occupy lowest unoccupied molecular orbital levels of the nucleobases as detected with electron spin resonance and x-ray absorption near edge structure measurements. Delocalization and strong correlations between the unpaired electrons are evident from a temperature-independent spin susceptibility and a microwave conductivity above $100 \mathrm{~K}$.
\end{abstract}

DOI: 10.1103/PhysRevLett.104.156804

PACS numbers: 36.20.Kd, 73.90.+f, 87.14.gk, 87.15.Pc

DNA has been considered as a potentially conductive material since the 1960s when Eley and Spivey [1] made their first conductance measurements on bulk DNA samples. In the late 1990s with a development of nanoscience and nanotechnology a research activity on DNA electronic properties sharply increased. The unique properties of DNA single strands - complementarity and recombination ability - would enable formation of complex nanocircuits by self-assembly and make DNA an important material in nanoelectronics. But at that time it was not clear if DNA is a sufficiently good conductor to be used in nanoelectronics. Early two-contact measurements of conductivity on single molecules or bundles of few DNA molecules gave discrepant results which spanned the whole spectrum of conductivities from insulating [2] and semiconducting [3] to conducting [4] (for a review, see Ref. [5]). Reasons for that had been sought in different experimental conditions [6]. However, it was shown lately that even in well controlled experiments conducted on short, artificial DNA with uniform sequence of base pairs and well-controlled electrical contacts, DNA is a very poor conductor of electricity [7]. This is not surprising if we invoke an analogy with quasi-1D organic conductors. Both DNA and the 1D conductors consist of planar stacks of heterocyclic aromatic molecules with intrastack distances of $\sim 3 \AA$, but in the case of DNA we lack counterions which would partially fill a conduction band of the 1D $\pi-$ $\pi$ stack. DNA has a valence band composed of nucleobases HOMO (highest occupied molecular orbital) orbitals and a conduction band composed of nucleobases LUMO (lowest unoccupied molecular orbital) orbitals. They are separated by an energy gap of $\sim 4 \mathrm{eV}$ causing the macromolecule to be an insulator. Therefore, in order to improve conducting properties of DNA one should add either additional electrons in the conducting band or additional holes in the valence band. A possibility of electron doping of DNA emerged with discovery of $M$-DNA [8], a complex which DNA spontaneously forms with transition metal cations, $\mathrm{Co}^{2+}, \mathrm{Ni}^{2+}$, and $\mathrm{Zn}^{2+}$, in buffered water solutions at high $p \mathrm{H}$ ( $\geq 8.5$ ). Fluorescence quenching [9] and electrochemical measurements [10] in solution have shown a strong increase in electron transfer rates upon formation of the $M$-DNA complex. Until now there has been only one measurement of $M$-DNA electric conductivity in the solid state [11]. Measured $I-V$ characteristics for native DNA and $M$-DNA have shown significant differences between a semiconducting behavior for the former and a metalliclike behavior for the latter. But, the mechanism of improved conductivity or even metallic properties was not clear. In order to detect free charge carriers in $M$-DNA Mizoguchi et al. [12] performed ESR measurements on the Zn-DNA complex and obtained a result which apparently contradicted the conductivity measurements. At that point it was not clear if any chemical doping takes place in the formation of $M$-DNA. In this Letter we will show that it is possible to obtain solid $M$-DNA complexes which show a high degree of electron doping by using a proper sample preparation procedure.

In our experiments we used native calf thymus DNA, sodium salt (Sigma). DNA was dissolved in a $10 \mathrm{mM}$ Tris$\mathrm{HCl}$ buffer solution, $p \mathrm{H} 9$ in concentration $100 \mu \mathrm{g} / \mathrm{ml}$. A $1 \mathrm{M}$ solution of either $\mathrm{ZnCl}_{2}$ or $\mathrm{Zn}\left(\mathrm{ClO}_{4}\right)_{2}$ was added to the DNA solution to obtain a final $\mathrm{Zn}^{2+}$ concentration of $1.5 \mathrm{mM}$. Instead of using a drying procedure by precipitation with cold ethanol described in Ref. [12], we have used a freeze-drying method to extract a solid material from the solution [13]. A resulting dry, fluffy material is composed mostly of recrystallized Tris-HCl buffer with about $7 \%$ of the Zn-DNA material. For the low temperature ESR measurements we sealed $\sim 30 \mathrm{mg}$ of the material under vacuum in a standard quartz ESR tube. For microwave measurements the samples were prepared at ambient con- 
ditions. For the $X$-band $(9.6 \mathrm{GHz}) \mathrm{cw}$-ESR experiments a homebuilt ESR spectrometer was equipped with a Varian E-101 microwave bridge, a Varian rectangular TE102 resonant cavity, and an Oxford Cryogenics E900 continuous-flow helium cryostat. The microwave conductivity was measured with a cavity perturbation technique [14] where the sample was positioned at the maximum electric field of $\mathrm{TE}_{011}$-mode cylindrical cavity with resonance frequency of $16.3 \mathrm{GHz}$. The $\mathrm{Zn} K$-edge XANES data of the Zn-DNA complex samples and reference $\mathrm{Zn}$ compounds were obtained in a standard transmission mode at the $\mathrm{C}$ station of HASYLAB synchrotron facility, DESY (Hamburg, Germany).

A typical room-temperature ESR spectrum of the freezedried Zn-DNA is shown in Fig. 1. It is strong, very broad and asymmetric. A calibration of the integrated ESR intensity with $\mathrm{CuSO}_{4} \cdot 5 \mathrm{H}_{2} \mathrm{O}$ [15] has shown that a number of spins in the samples vary from about 0.2 to almost 1 spin per base pair (bp) of DNA, where 1 spin per bp is the upper limit determined with the stoichiometry of Zn-DNA. The linewidth of the ESR signal also varies from sample to sample with a mean variance around $200 \mathrm{G}( \pm 30 \mathrm{G})$. A line of similar width is observed in $Q$-band ESR (12 kG field) [16], suggesting that inhomogeneous broadening is not significant. The line shape of the ESR signal is asymmetric but could be fitted well with a Lorentzian curve with a slight contribution of dispersion [17] (see Fig. 1). The empirical asymmetry parameter $A / B=1.5$ does not vary significantly for different samples, implying that the asym-

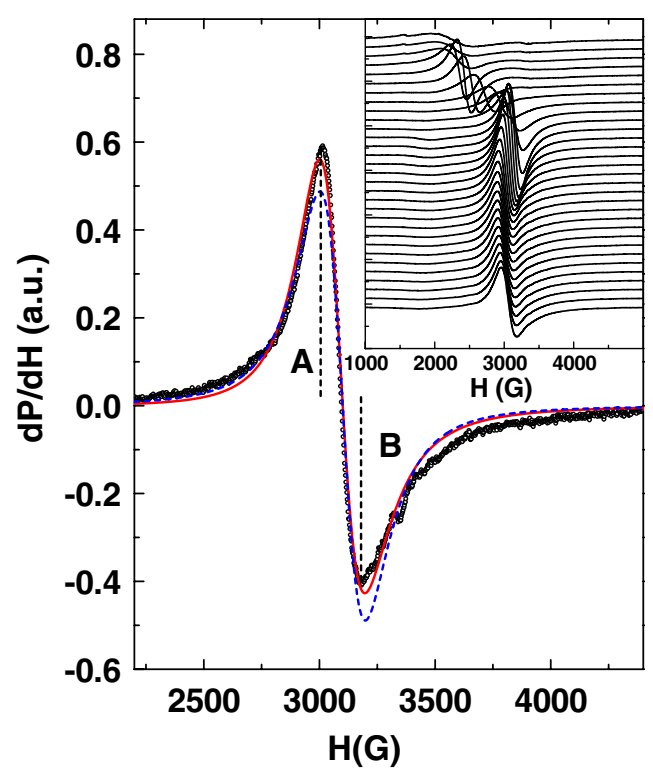

FIG. 1 (color online). The room-temperature ESR spectrum of Zn-DNA (black circles) fitted with an asymmetric Lorentzian (Dysonian) (solid red line) a Lorentzian (dashed blue line) curves. The inset shows a temperature development of the $\mathrm{Zn}$ DNA ESR resonance measured on cooling from a room temperature (the bottom curve) down to $10 \mathrm{~K}$ (the top curve) measured at every $10 \mathrm{~K}$. metry is intrinsic. Though our bulk samples hardly satisfy the Dyson's criterion for the asymmetric resonant line [18], some microscopic parts of the samples could have a conductivity which is high enough to produce the asymmetric resonant line like in the case of low-doped polymers [19]. Another possibility for an asymmetric resonant line could be also the admixture of nondiagonal elements of dynamic susceptibility to the ESR signal as observed in some linearchain magnets [20]. The ESR resonance is centered at a $g$ value $\sim 2.2$ which differs significantly from the free electron value, 2.0023. The detected ESR signal is orders of magnitude larger that any signal which could originate from paramagnetic impurities. Still, to eliminate extrinsic origins for the signal, three control samples with the same freeze-drying method and with the same starting chemicals were prepared: (a) tris- $\mathrm{HCl}+\mathrm{ZnCl}_{2}+\mathrm{DNA}$ at $p \mathrm{H} \mathrm{7}$, (b) tris- $\mathrm{HCl}+\mathrm{ZnCl}_{2}$ at $p \mathrm{H} \mathrm{9}$, and (c) tris- $\mathrm{HCl}+\mathrm{DNA}$ at $p \mathrm{H}$ 9. None of them is expected to form the Zn-DNA complex and none of them shows any ESR signal. Importantly, the control sample (b), which mimics the excess $93 \%$ buffer (salt) matrix in our Zn-DNA samples, shows no signal, so we can conclude that the unpaired electrons reside on the Zn-DNA complex.

In the inset of Fig. 1 we show the temperature dependence of the ESR resonance line. It is nearly $T$ independent between room temperature and $100 \mathrm{~K}$. Below $100 \mathrm{~K}$ the line abruptly broadens and shifts towards lower magnetic fields. The temperature dependences of the spin susceptibility $\chi_{\text {spin }}$, the linewidth $\Delta H$, and the $g$ value extracted directly from the resonance curves are shown in Figs. 2(a)2 (c), respectively. Besides a broad local maximum in $\chi_{\text {spin }}$ and $g$ value centered at $200 \mathrm{~K}$ and a slight decrease in $\Delta H$ below $200 \mathrm{~K}$, all three parameters are fairly constant in the high temperature interval between 100 and $300 \mathrm{~K} . \chi_{\text {spin }}$ is almost constant and does not follow a Curie-Weiss law [see the inset of Fig. 2(a)] which might be expected for a system of localized spins. Below $100 \mathrm{~K}$ the $g$ value starts to continuously increase with decreasing temperature suggesting a development of an internal local magnetic field.

To evaluate the conductivity $\sigma_{\mathrm{MW}}$, we use the scheme of a depolarization regime [14], where the microwave penetrates throughout the sample. The absolute value of conductivity is calculated for the bulk sample and contains about $30 \%$ error. Nearly $T$-independent behavior is shown between 100 and $220 \mathrm{~K}$ (Fig. 3), which is common in highly correlated electron systems [21]. Above $220 \mathrm{~K}$, $\sigma_{\mathrm{MW}}$ shows a steep increase. A similar increase was also observed in Na-DNA where it was attributed to the rotational motion of water molecules hydrated to the negatively charged phosphate groups [22,23]. Below $100 \mathrm{~K}$, where $\chi_{\text {spin }}$ and $\Delta H$ show a pronounced cusp indicating a transitionlike behavior, a small but clear step of the microwave (MW) conductivity is observed (Fig. 3). While no proper phase transition can be expected in Zn-DNA because the linear 1D DNA molecules are dispersed in a 


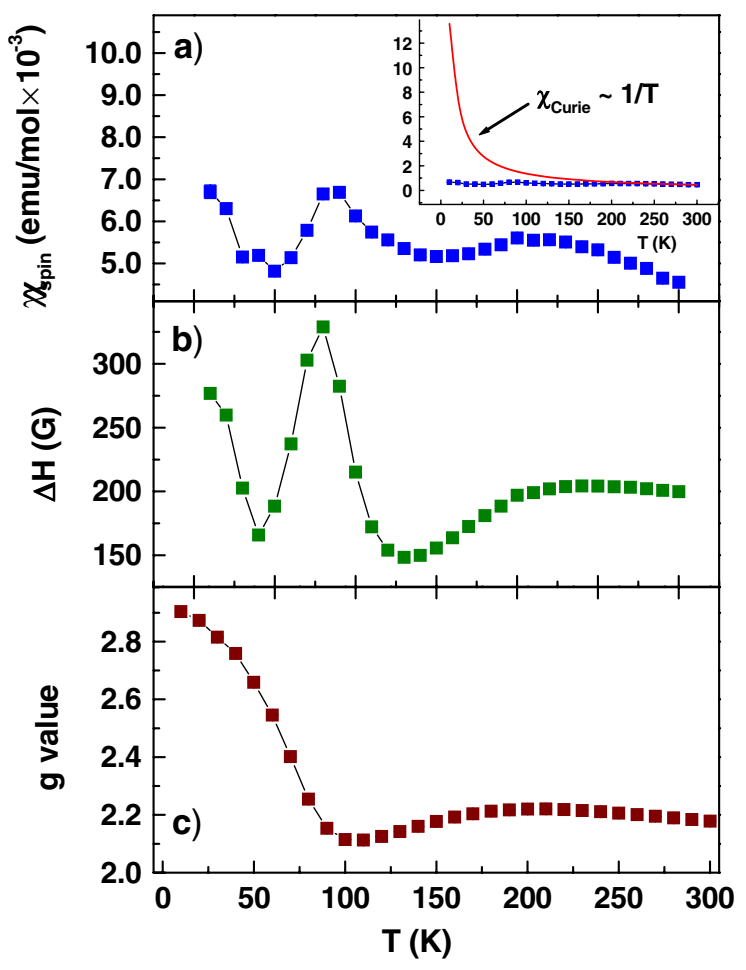

FIG. 2 (color online). Temperature dependences of (a) a spin susceptibility $\chi_{\text {spin }}$, (b) a resonance line width $\Delta H$, and (c) a $g$ value. The inset of (a) shows comparison of the temperature dependence of the spin susceptibility of Zn-DNA with the Curie law on an expanded scale. The susceptibility is expressed in mol of DNA base pairs.

recrystallized buffer and salt matrix, so there is no possibility of intermolecular 3D interactions that could lead to long range order. Nevertheless we can still expect short range $1 \mathrm{D}$ correlations between unpaired spins along segments of individual DNA molecules. At the lowest temperatures $(<50 \mathrm{~K})$ the $\mathrm{MW}$ conductivity goes to zero and the spin susceptibility increases-both indicating electron localization.

In contrast to some previous ESR studies on DNA [24] where a very low level of electron doping was realized by

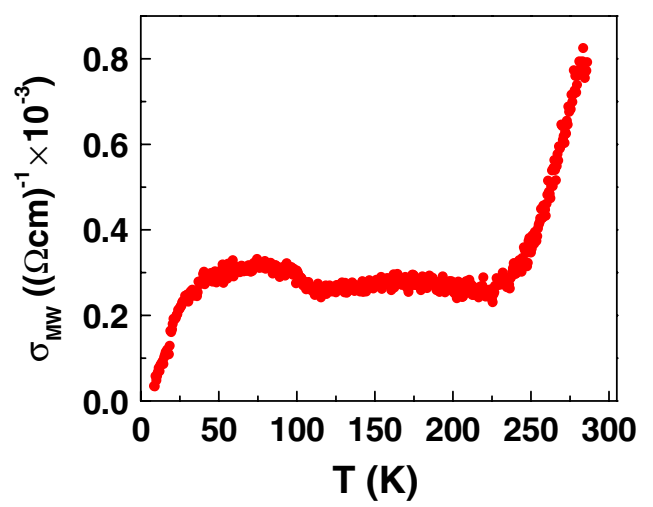

FIG. 3 (color online). A temperature dependence of the MW conductivity of Zn-DNA. irradiation of glassy matrices at $77 \mathrm{~K}$, in the Zn-DNA complex we obtain a very high level of electron doping, so electron-electron correlations become important. Generally, the $M$-DNA complex forms when divalent metal cations, $\mathrm{M}^{2+}$ replace imino protons, $\mathrm{H}^{+}$from the hydrogen bonds between complementary nucleobases in the core of the double helix, as confirmed by ${ }^{1} \mathrm{H}$ NMR [8]. This leads to a net positive charge on the DNA molecule, which is effectively neutralized in a buffered solution with high salt concentration. When the complex is dried, its net positive charge cannot be simply neutralized with negative ions on the outer surface of the double helix because this would cost too much electrostatic energy. Apparently, much like in the irradiated DNA samples, free electrons from the frozen matrix penetrate the double helix to ensure local charge neutrality. There are two possibilities for the location of unpaired electrons inside the double helix: on the zinc cations or on the nucleobases. The first possibility involves a reduction of a diamagnetic $\mathrm{Zn}^{2+}$ with the electronic structure $4 s^{0} 3 d^{10}$ to a paramagnetic $\mathrm{Zn}^{+}$with the electronic structure $4 s^{1} 3 d^{10}$. In order to check for $\mathrm{Zn}^{+}$ions (which are indeed very rare in nature [25]) in Zn-DNA we have performed $\mathrm{x}$-ray absorption near edge structure (XANES) measurements of the zinc $K$ edge on the $\mathrm{Zn}$ DNA complex and control samples. The average $\mathrm{Zn}$ valence in the samples can be obtained from the energy position of the $\mathrm{Zn} K$ edge. The valence is known to affect the position of the absorption edge [26], which shifts towards higher energies with increasing oxidation. For atoms with the same type of ligand a linear relation between the edge shift and the valence state was established. The Zn XANES spectra of Zn-DNA complexes and the reference compounds have shown no differences in the position of the $\mathrm{Zn}$ absorption edge of the samples with and without intercalated zinc ions [16]. The edge position coincides with that in $\mathrm{ZnCl}_{2}$ compound, which clearly indicates that in all samples the oxidation state of the zinc ions is +2 (smaller amounts of $\mathrm{Zn}$ in another valence state of the order of few percent cannot be excluded). Therefore, we must accept that the unpaired electrons are located on the DNA nucleobases.

The ESR spectra of the reduced forms of DNA nucleobases are well documented in the literature [27]: singlets for $A^{-}$and $G^{-}$and doublets for $C^{-}$and $T^{-}$with linewidths between 20 and $30 \mathrm{G}$. They were measured on isolated spins at low temperatures. In our case, the ESR signal of Zn-DNA has a linewidth which is about 10 times larger. Since in organic systems the main line-broadening mechanism is via electron-electron dipolar interactions we attribute the large linewidth to the high spin concentration in $\mathrm{Zn}$ DNA. In order to estimate the dipolar contribution to the ESR linewidth we assumed that $S=1 / 2$ states are separated at a typical distance of $r=3.4 \AA$ between neighboring bases. By performing a powder average of the dipolar second moment $M_{2}^{\text {dip }}=\frac{3}{4} S(S+1) \mu_{B}^{2} g^{4} \sum \frac{\left(3 \cos ^{2} \vartheta-1\right)^{2}}{r^{6}}$, we es- 
timate the dipolar linewidth $\Delta H=\sqrt{M_{2}} \approx 450 \mathrm{G}$, which is in a reasonable agreement with our measurements.

The direction of the $g$-value shift in microscopically disordered samples with low or no intrinsic magnetic anisotropy can tell us the sign of the spin-spin correlations [28]. The shift is a direct consequence of dipolar interactions between unpaired spins. For randomly oriented spins the dipolar field averages to zero, but for ferromagnetically correlated spins with preferred orientation in a direction of the external applied field $H_{\text {dipol }}^{z}=\left\langle\mu^{z}\right\rangle\left(3 \cos ^{2} \vartheta-1\right) / r^{3}$, assuming a zero anisotropy in the system itself (i.e., $\langle\boldsymbol{\mu}$. $\mathbf{r}\rangle=\langle\boldsymbol{\mu}\rangle \cdot\langle\mathbf{r}\rangle$ ). Hence, $\left\langle\mathbf{H}_{\text {dipol }}^{i}\right\rangle \neq 0$ and is positive (in a direction of the external field). The external and the dipolar fields add to give a resonant field: $H_{\text {ext }}+\left\langle H_{\text {dipol }}\right\rangle=H_{\text {res }}$, and consequently, the ESR line moves towards lower applied fields for a given MW frequency $\omega$. For systems with nondegenerate orbitals, such as Zn-DNA, the magnetic correlations are typically antiferromagnetic [29], but nevertheless, several 1D organic radical systems such as ferromagnetic $p$-nitrophenyl nitronyl nitroxide ( $p$-NPNN) [30] with ferromagnetic interactions between unpaired spins are well known.

In conclusion, we have shown that by a freeze-drying method we could extract a solid metal-DNA complex ZnDNA which shows many characteristics of strongly correlated 1D electron systems. The 1D electron system is realized by transferring electrons to empty LUMO levels of DNA nucleobases. The electrons are not localized above $100 \mathrm{~K}$, in agreement with recent results of soft x-ray spectroscopy [31], and start to localize only at low temperatures below $50 \mathrm{~K}$. Apparently, the realization of high electron density in the conduction band of DNA by electron doping in the metal-DNA complex, which is essential for any future application of $M$-DNA in nanoelectronics, also introduces the physics of strongly correlated systems into the field of doped DNA.

This work has been supported by Slovenian Research Agency research programs P10040 and P1-011 and by DESY, and the EC under FP6 Program "Structuring the European Research Area" contract RII3-CT-2004-506008 (IA-SFS). We would like to thank E. Welter (HASYLAB) and Dr. P. Cevc (ESR, J. Stefan Inst.) for their assistance.

[1] D. D. Eley and D. I. Spivey, Trans. Faraday Soc. 58, 411 (1962).

[2] P. J. de Pablo et al., Phys. Rev. Lett. 85, 4992 (2000).

[3] D. Porath et al., Nature (London) 403, 635 (2000).
[4] H. W. Fink and C. Schonenberger, Nature (London) 398, 407 (1999).

[5] R. G. Endres, D. L. Cox, and R. R. P. Singh, Rev. Mod. Phys. 76, 195 (2004).

[6] D. Porath, G. Cuniberti, and R. Di Felice, Top. Curr. Chem. 237, 183 (2004).

[7] A. J. Storm, J. van Noort, S de Vries, and C. Dekker, Appl. Phys. Lett. 79, 3881 (2001); C. Gomez-Navarro et al., Proc. Natl. Acad. Sci. U.S.A. 99, 8484 (2002).

[8] J. S. Lee, L. J. P. Latimer, and R. S. Reid, Biochem. Cell Biol. 71, 162 (1993).

[9] P. Aich et al., J. Mol. Biol. 294, 477 (1999).

[10] C.-Z. Li et al., J. Phys. Chem. B 107, 2291 (2003); Y.-T. Long et al., Biophys. J. 84, 3218 (2003).

[11] A. Rakitin et al., Phys. Rev. Lett. 86, 3670 (2001).

[12] K. Mizoguchi et al., Phys. Rev. B 72, 033106 (2005).

[13] $50 \mathrm{ml}$ of the solution was first incubated for $2 \mathrm{~h}$ at room temperature and then transferred into a test tube which was precooled with liquid nitrogen. The test tube was then immersed in a Dewar flask filled with ice and connected to a vacuum system. The freeze drying of $50 \mathrm{ml}$ of solution is usually completed in 3 days.

[14] O. Klein, S. Donovan, M. Dressel, and G. Gruner, Int. J. Infrared Millim. Waves 14, 2423 (1993).

[15] In estimating the number of unpaired spins in our samples we used an approximation of localized spins and a formula for susceptibility $\chi=N \mu^{2} / k_{B} T$, where $T$ is a room temperature. $T$ differs from some unknown $T_{\text {eff }}$, which would be proper for the correlated electronic system but even that $T_{\text {eff }}$ cannot exceed $\sim 0.1 \mathrm{eV}$ which is a conduction bandwidth for DNA. Thus, the estimated doping level in $\mathrm{Zn}-\mathrm{DNA}$ is accurate to at least an order of magnitude.

[16] See supplementary material at http://link.aps.org/ supplemental/10.1103/PhysRevLett.104.156804 for the $Q$-band ESR $(34.9 \mathrm{GHz})$ resonance line and x-ray absorption near edge structure (XANES) spectra of Zn-DNA and the reference compounds.

[17] Z. Seidov et al., Phys. Rev. B 65, 014433 (2001).

[18] F. J. Dyson, Phys. Rev. 98, 349 (1955).

[19] P. Bernier et al., Synth. Met. 17, 413 (1987).

[20] H. Benner et al., J. Phys. C 16, 6011 (1983).

[21] Y. Taguchi et al., Phys. Rev. B 48, 511 (1993).

[22] A. Hojo et al., J. Phys. Soc. Jpn. 77, 044802 (2008).

[23] H. Matsui et al., Phys. Rev. B 79, 235201 (2009).

[24] A. Messer et al., J. Phys. Chem. B 104, 1128 (2000).

[25] Y. Tian, G.-D. Li, and J.-S. Chen, J. Am. Chem. Soc. 125, 6622 (2003).

[26] J. Wong et al., Phys. Rev. B 30, 5596 (1984).

[27] W. A. Bernhard, J. Phys. Chem. 93, 2187 (1989).

[28] A. Fainstein, J. Phys. Condens. Matter 4, 4989 (1992); V. Tangulis et al., Dalton Trans. 1752 (2008).

[29] A. J. Epstein et al., Phys. Rev. B 5, 952 (1972).

[30] M. Takahashi et al., Phys. Rev. Lett. 67, 746 (1991).

[31] J. B. McNaughton et al., Phys. Rev. B 73, 205114 (2006). 